\section{Nachruf auf Joachim Zerdick}

\section{Obituary Joachim Zerdick}

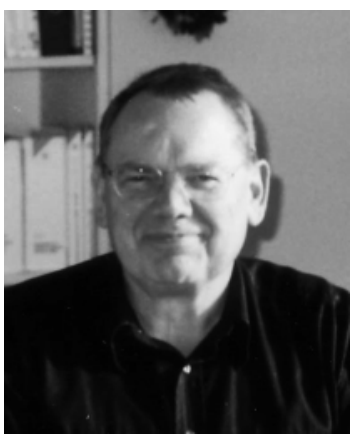

Dr. med. Joachim Zerdick

Am 20.1.2004 verstarb nach schwerer Krankheit im Alter von 60 Jahren Dr. med. Joachim Zerdick.

Joachim Zerdick war viele Jahre Vorstandsmitglied der Deutschen Gesellschaft für Suchtmedizin (vorm. DGDS) e.V. Er hatte von 1995-2000 die jährlichen suchtmedizinischen Kongresse organisiert. Wichtig war für ihn dabei die Unabhängigkeit von der pharmazeutischen Industrie bei der Themenauswahl. Er beherrschte den schwierigen Balanceakt, diese Unabhängigkeit zu bewahren und gleichzeitig die Kongresse solide zu finanzieren. Mit großem organisatorischen Geschick gelang es ihm, die
Kongressbände rechtzeitig zum Kongressbeginn fertig stellen zu lassen.

Er war seit 1986 als beratender Arzt in der Bezirksstelle Hannover der Kassenärztlichen Vereinigung tätig. Er hat über all die Jahre die Versorgung von Suchtkranken zu einem seiner Betrachtungsschwerpunkte gemacht. Auf seine Initiative hin und mit seiner beharrlichen Mitwirkung ist in Hannover ein bundesweit beachtetes Modellprojekt zur Drogensubstitution in Form der Praxis für Drogenkranke entstanden.

Daneben war er in Hannover im Vorstand der Beratungsstelle Prisma e.V. sowie im Fachbeirat der Drogentherapiestation für Jugendliche Teen Spirit Island tätig. Zudem leitete den Qualitätszirkel „Substitutionstherapie Opiatabhängiger“.

Als Vorstandsmitglied der DGS und als beratender Arzt bei der KV-Bezirksstelle Hannover hat er dazu beigetragen, dass Suchtkranke in ärztlichen Praxen qualifiziert behandelt werden.

Wir haben sehr gerne mit Joachim Zerdick zusammengearbeitet. 